Pacific Journal of Mathematic 


\title{
COMPLETION OF MATHEMATICAL SYSTEMS
}

\author{
A. H. KRUSE
}

1. Introduction. The completion problem to be considered may be informally and tentatively described as follows.

Let $\mathscr{A}$ be a class of systems of some type (e.g., $\mathscr{A}$ will be the class of all fields in Example 1; cf. $\S \S 6,7)$. For all $a, b \in \mathscr{A}$ let " $a \prec b$ " mean that $a$ is a subsystem (e.g., subfield in Example 1) of $b$. For each $a \in \mathscr{A}$ let $\pi(a)$ be a set of propositional forms ${ }^{1}$ involving unknowns (e.g., polynomial equations in one unknown in Example 1); each of these forms may become a true or false proposition upon substitution of elements of $a$ for the unknowns; a substitution turning a form into a true proposition is a solution of the form. For each $a \in \mathscr{A}$ let $\pi^{\prime}(a)$ be the set of all members of $\pi(a)$ with solutions (relative to $a$ ). If $a, b \in \mathscr{A}$ and $a \prec b$, then each $p \in \pi(a)$ will correspond to some member, say $\rho_{a}^{b}(p)$, of $\pi(b)$ (e.g., if $\mathscr{A}$ is the class of all groups, the propositional form " $y^{-1} x y \neq x$ for some $y$ in $a$ " in unknown $x$ could correspond to " $y^{-1} x y \neq x$ for some $y$ in $b$ "). We may say that $a \in \mathscr{A}$ is complete if and only if for each $b \in \mathscr{A}$ with $a \prec b$ and each $p \in \pi(a)$ : if $p$ has no solution (relative to $a$ ), then $\rho_{a}^{b}(p)$ has no solution (relative to $b$ ). (E.g., in Example 1, a field is complete if and only if it is algebraically closed.) The completion problem to be considered is: Does each $a \in \mathscr{A}$ have a complete extension? ${ }^{2}$

This extension problem will be formulated rigorously in $\S \S 5,6$. In some explicit special cases in modern algebra the existence of a complete extension rests on (transfinitely) recursive definitions the justification of which at first glance would seem to require a very strong version of the axiom of choice (cf. Remark 5 of $\S 7$ ). In this paper the set-theoretic foundations of such procedures will be examined. The result is a theorem from which will follow the usual extension theorems via the usual weak version of the axiom of choice.

2. Set-theoretic preliminaries. In axiomatic set theory one may consider the following versions of the axiom of choice.

Received July 15, 1959, and in revised form August 7, 1961.

(A) Earlier versions of this paper consisted of research done at the University of Kansas partially supported by National Science Foundation Grant NSF-G 4917. The writer is indebted to W. R. Scott for reading the first version and making several suggestions. The writer is indebted to the referee for a number of comments and suggestions, some leading to substantial improvements over earlier versions of this paper.

1 Relative to (axiomatic) set theory, since a propositional form exists only in the metatheory, the propositional forms as such will have to be replaced by set-theoretic antecedents.

2 This completion problem is a straightforward generalization of problems raised and solved by W. R. Scott [6] for groups (however, cf. Remark 5 of §7). The general problem of this paper will be illustrated by Scott's result via Example 2 (cf. §§6, 7). 
Weak version. If $M$ is a set of nonvoid sets, there is a function $\varphi$ on $M$ such that $\varphi(X) \in X$ for each $X \in M$.

Strong version. If $\mathscr{M}$ is a class ${ }^{3}$ of nonvoid sets, there is a function $\varphi$ on $\mathscr{C}$ such that $\varphi(X) \in X$ for each $X \in \mathscr{M}$.

Stronger version. If $\mathscr{C}$ is a class, there is a function $\varphi$ on $\operatorname{dom}(\mathscr{C l})^{4}$ such that $\varphi \subset \mathscr{M} .^{5}$

Strongest version. If $\mathscr{C}$ is a class, there is a function $\varphi$ on $\operatorname{dom}(\mathscr{C})$ such that $\varphi \subset \mathscr{C}$ and such that for all $u, v \in \operatorname{dom}(\mathscr{M})$, if for each object $y,[u, y] \in \mathscr{C}$ if and only if $[v, y] \in \mathscr{L}$, then $\varphi(u)=\varphi(v){ }^{6}$

In the rest of this paper we will assume that we are working in a set theory of the kind considered by Mostowski [4, pp. 4-6] with the Fundierungsaxiom not assumed and with the weak version of the axiom of choice assumed. Such a set theory may be obtained by modifying the system considered by Gödel [3] (this system being essentially developed by von Neumann and further refined by Bernays [1]) as follows. There is allowed the existence of atoms (also called Urelements) - elements which are not sets - , and the usual axioms are modified to accomodate them. Thus each object in the theory is either an element or a class. A set is an element which is a class. An atom is an element which is not a class. Intuitive language will be used throughout, but with precision.

We now have what might be regarded as a weakest reasonable set theory in which practically all of modern mathematics can be developed. In considering such a minimal set theory the writer is interested in generality not only as such but also in its effects on foundational methodology.

The writer feels (without proof) that the stronger version of the axiom of choice is not a consequence of the strong version in the theory under consideration. The stronger version has been stated partly in anticipation of Remark 5 of $\S 7$.

Throughout this paper $\mathcal{O}$ will be the class of all ordinal numbers. ${ }^{7}$ The void set is $0 \in \mathcal{O}$.

For each class $\mathscr{K}, \mathscr{P}(\mathscr{C})$ will be the class of all subsets of $\mathscr{K}$.

3 A class may be too big to be a set.

${ }^{4}$ For each class $\mathscr{M}$, the domain of $\mathscr{H}$ is the class dom $(\mathscr{K})$ consisting of all $x$ for which $[x, y]=\{\{x\},\{x, y\}\} \in \mathscr{M}$ for some $y$, and the image of $\mathscr{K}$ is the class $\operatorname{im}(\mathscr{K})$ consisting of all $y$ for which $[x, y] \in \mathscr{M}$ for some $x$.

5 The stronger version says roughly that there is a choice function for every "family" of classes, the "family" of classes being " $\{\{y \mid[x, y] \in \mathscr{M}\}\}_{x \in \operatorname{dom}(\mathscr{M})}$ ".

${ }_{6}$ The strongest version says roughly that there is a choice function for every "class" of classes, the "class" of classes being " $\{\{y \mid[x, y] \in \mathscr{M}\} \mid x \in \operatorname{dom}(\mathscr{M})\}$ ". In the von NeumannBernays-Gödel theory including the axiom of foundation (and in which every member of each class is a set) the strongest version is a consequence of the strong version via wellordering of the universe (cf. [8] and [1, pp. 70-71]).

7 Each ordinal number consists of its predecessors. A cardinal number is an ordinal number not equi-potent with any of its members. 
For each set $X$, we define $\mathscr{P}^{\beta}(X)$ for each $\beta \in \mathscr{O}$ so that $\mathscr{P}^{0}(X)=X$, $\mathscr{P}^{\beta+1}(X)=\mathscr{P}\left(\mathscr{P}^{\beta}(X)\right)$ for each $\beta \in \mathcal{O}$, and

$$
\mathscr{P}^{\beta}(X)=\bigcup\left\{\mathscr{P}^{\alpha}(X) \mid \alpha<\beta\right\}
$$

for each nonzero limit ordinal number $\beta$.

Suppose $X$ and $Y$ are sets of atoms. Then for each

$$
x \in\left(\bigcup\left\{\mathscr{P}^{\alpha}(X) \mid \alpha \in \mathscr{O}\right\}\right) \cap\left(\bigcup\left\{\mathscr{P}^{\alpha}(Y) \mid \alpha \in \mathscr{O}\right\}\right)
$$

and each $\gamma \in \mathcal{O}, x \in \mathscr{F}^{\gamma}(X)$ if and only if $x \in \mathscr{P}^{\gamma}(Y)$ (by induction on $\gamma$ ). From this it follows (by induction on $\gamma$ ) that for each nonvoid class $\mathscr{M}$ of sets of atoms and each $\gamma \in \mathcal{O}$,

$$
\mathscr{P}^{\gamma}(\bigcap \mathscr{C})=\bigcap\left\{\mathscr{P}^{\gamma}(X) \mid X \in \mathscr{M}\right\} .
$$

It now follows that for each element $x$, if there is a set $X$ (perhaps void) of atoms such that $x \in \mathscr{P}^{\gamma}(X)$ for some $\gamma \in \mathcal{O}$, then there is a minimum such set $X$. Such a minimum set $X$ will be called a foundation of $x$.

Although we will not assume it without specific mention, we shall have occasion to consider the effects of the axiom of foundation (Fundierungsaxiom): If $\mathscr{C}$ is a nonvoid class of sets, then $\mathscr{C} \cap X$ is void for some $X \in \mathscr{M}$. In the system of set theory in which we are working the axiom of foundation is equivalent to the (in the writer's opinion, intuitively better motivated) statement: For each set $X, X \in \mathscr{P}^{\gamma}(A)$ for some $\gamma \in \mathcal{O}$ and some set $A$ of atoms. ${ }^{8}$ Thus the axiom of foundation is equivalent to the statement that each set has a foundation.

Consider a set $X$. Now $\cup X=\{u \mid u \in x$ for some $x \in X\}$. We define $\bigcup^{\alpha} X$ for each ordinal number $\alpha \leqq \omega^{9}$ by induction so that $\bigcup^{0} X=$ $X, \bigcup^{\alpha+1} X=\bigcup^{\alpha} X$ for each $\alpha \in \omega$, and $\bigcup^{\omega} X=\bigcup\left\{\bigcup^{\alpha} X \mid \alpha \in \omega\right\}$. Now $\mathrm{U}^{\omega} X$ is the transitive closure of $X$ as defined in [1, IV, p. 136] (cf. also [1, VI, p. 68]), i.e., the smallest set $Y$ such that $X \subset Y$ and such that $Z \subset Y$ for each set $Z \in Y$. Let $A=\left\{x \mid x \in \bigcup^{\omega} X ; x\right.$ is an atom $\}$. It may be shown that $X$ has a foundation if and only if $X \in \mathscr{P}^{\gamma}(A)$ for some $\gamma \in \mathcal{O}$. It may be shown that if $X$ has a foundation, then $A$ is the foundation of $X$.

3. Subuniverses. The content of $\S 3$ remains valid in the absence of an axiom of choice. We define a subuniverse to be any class $\mathscr{V}$ such that for each set $X, X \in \mathscr{Y}$ if and only if $X \subset \mathscr{Y}$.

Suppose $\mathscr{Y}$ is a subuniverse. Recall that all notions within the theory may be defined in terms of the primitive notions element, class,

8 This may be shown by making obvious modifications in the discussion in [1, VI, §16]. There the function $\Psi$ is such that $\Psi(\alpha)=\mathscr{P}^{\alpha}(0)$ for each $\alpha \in 0$.

${ }^{9} \omega$ is the first infinite ordinal number. 
$\epsilon$ by use of the usual logical connectives and the notion of logical identity (these are formalized in the first order predicate calculus with equality). If the notions element, class, $\Phi \in \Psi$ are replaced by the notions member of $\mathscr{V}^{\prime}$, subclass of $\mathscr{Y}, \Phi \in \Psi \subset \mathscr{Y}$ respectively and all subsequent notions are modified accordingly, the axioms of set theory give rise to new statements which may be proved from the original axioms and the statement that $\mathscr{Y}$ is a subuniverse. These observations motivate the choice of the term subuniverse. (Cf. § 8.)

Suppose $\mathscr{Y}^{-}$is a subuniverse. It may be proved by transfinite induction that $\gamma \in \mathscr{Y}$ for each $\gamma \in \mathcal{O}$. Thus $\mathscr{O} \subset \mathscr{Y}$. Moreover, if $X \in \mathscr{Y}$, then $\mathrm{U}^{\omega} X \in \mathscr{Y}$. If $X \in \mathscr{Y}$, then $\mathscr{P}^{\alpha}(X) \in \mathscr{Y}$ for each $\alpha \in \mathbb{C}^{\circ}$. Under the switch in terminology described in the previous paragraph, ordinal number is invariant. The same is true of the "operators" $\mathrm{U}^{\alpha}(\alpha \leqq \omega)$ and $\mathscr{P}^{\alpha}\left(\alpha \in \mathcal{O}^{\prime}\right)$ when they are restricted to members of $\mathscr{V}$. The same is true of many other notions, and this point will be belabored no further here. (Cf. $\S 8$.)

From now on, $\mathscr{U}$ will be the universe (thus $\mathscr{U}$ is the largest subuniverse), and $\mathscr{U}_{0}$ will be the class of all atoms.

For each class $\mathscr{C l}$ we define the hull of $\mathscr{A}$ to be the class

$$
\mathscr{H}(\mathscr{A})=\bigcup\left\{\mathscr{P}^{\alpha}\left(\bigcup^{\omega} X\right) \mid[X, \alpha] \in \mathscr{P}(\mathscr{A}) \times \mathscr{O}\right\} .
$$

For each atom $a$ we define the hull of $a$ to be the class $\mathscr{H}(a)=$ $\mathscr{H}(\{a\})$. For each object $\Phi$ the hull of $\Phi$ is the smallest subuniverse of which $\Phi$ is either a subclass or a member and may be called the subuniverse generated by $\Phi$.

The axiom of foundation is equivalent to the statement that $\mathscr{U}=$ $\mathscr{H}\left(\mathscr{U}_{0}\right)$. This fact and the observations in the second paragraph of $\S 3$ constitute essentially the classical proof of the theorem that if the axiomatic system under consideration is consistent, then the axiomatic system constituted by the axioms of the system under consideration and the axiom of foundation is consistent; this proof is often given when $\mathscr{U}_{0}=0$, as in [1, VI], and is due to J. von Neumann [8]. Observe that $\mathscr{C}(0)$ is the smallest subuniverse.

4. Amenable classes. Given $\mathscr{C}$ and $\varphi$, we shall say that $\varphi$ makes $\mathscr{A l}$ amenable if and only if $\mathscr{C}$ is a class and $\varphi$ is a function on $\operatorname{dom}(\mathscr{C})$ and for each $x \in \operatorname{dom}(\mathscr{C}), \varphi(x)$ is a nonvoid set of elements $y$ such that $[x, y] \in \mathscr{M}$. Given $\mathscr{M}$ and $\varphi$, we shall say that $\varphi$ makes $\mathscr{A C}$ perfectly amenable if and only if $\varphi$ makes $\mathscr{C}$ amenable and for all $u, v \in \operatorname{dom}(\mathscr{C})$, if

$$
\{y \mid[u, y] \in \mathscr{C}\}=\{y \mid[v, y] \in \mathscr{C}\},
$$

then $\varphi(u)=\varphi(v)$. Given $\mathscr{L}$, we shall say that $\mathscr{C}$ is [perfectly] 
amenable if and only if for some $\varphi, \varphi$ makes $\mathscr{C l}$ [perfectly] amenable. Observe that if the class $\mathscr{C l}$ satisfies the condition in the stronger (resp., strongest) version of the axiom of choice, then $\mathscr{C}$ is amenable (resp., perfectly amenable). Observe that if $\mathscr{C l}$ is a class and $\{y \mid[x, y] \in \mathscr{C}\}$ is a set for each element $x$, then $\mathscr{C}$ is prefectly amenable. In particular, each set is perfectly amenable.

THeOREm 1. Suppose $\mathscr{A}$ is a class and each subclass of $\mathscr{H}(\mathscr{A}) \times$ $\mathscr{P}(\mathscr{A})$ is [perfectly] amenable. (Note. $\mathscr{H}(\mathscr{A}) \times \mathscr{P}(\mathscr{H}) \subset \mathscr{H}(\not)$.) Then each subclass of $\mathscr{\mathscr { C }}(\mathscr{A})$ is $\left[\right.$ perfectly] amenable..$^{10}$

Proof. Consider a class $\mathscr{C} \subset \mathscr{C}(\mathscr{A})$. Let $\mathscr{L}=\left\{[u, y] \mid u \in \mathscr{H}(\mathscr{A}) ; y \in \mathscr{P}(\mathscr{A}) ; u \in \mathscr{P}^{\gamma}\left(\bigcup^{\omega} y\right)\right.$ for some $\left.\gamma \in \mathscr{O}\right\}$.

There is a function, say $\psi$, which makes $\mathscr{L}$ [perfectly] amenable. Then $\operatorname{dom}(\psi)=\operatorname{dom}(\mathscr{L})=\mathscr{H}(\mathscr{A})$. Let

$$
\begin{array}{r}
\mathscr{N}=\{[x, y] \mid x \in \mathscr{C}(\mathscr{A}) ; \text { for some } u \in \mathscr{C}(\mathscr{A}),[x, u] \in \mathscr{C} \\
\text { and } y \in \psi(u)\} .
\end{array}
$$

Since $\mathscr{N} \subset \mathscr{H}(\mathscr{A}) \times \mathscr{P}(\mathscr{A})$, there is a function, say $\xi$, which makes $\mathscr{N}$ [perfectly] amenable. For each $x \in \operatorname{dom}(\mathscr{C})$, let $\beta(x)$ be the smallest $\gamma \in \mathcal{O}$ such that there are $u, y \in \mathscr{L}(\mathscr{A})$ for which $[x, u] \in \mathscr{L}$, $y \in \psi(u) \cap \xi(x)$, and $u \in \mathscr{P}^{\gamma}\left(\bigcup^{\omega} y\right)$ (the existence of such $\gamma$ follows from the definitions of $\xi, \mathscr{N}, \psi, \mathscr{L})$. For each $x \in \operatorname{dom}(\mathscr{L})$ let

$$
\begin{array}{r}
\varphi(x)=\{u \mid[x, u] \in \mathscr{C l} \text {; there is } y \in \psi(u) \cap \xi(x) \text { for which } \\
\left.\qquad u \in \mathscr{P}^{\beta(x)}\left(\bigcup^{\omega} y\right)\right\} .
\end{array}
$$

For each $x \in \operatorname{dom}(\mathscr{C})$,

$$
\varphi(x) \subset \bigcup\left\{\mathscr{P}^{\beta(x)}\left(\bigcup^{\omega} y\right) \mid y \in \xi(x)\right\}
$$

is a set. Now $\varphi=\{[x, \varphi(x)] \mid x \in \operatorname{dom}(\mathscr{C})\}$ makes $\mathscr{C}$ [perfectly] amenable. Q.e.d.

COROLlary 1.1. For each set $X$, each subclass of $\mathscr{H}(X)$ is perfectly amenable.

10 This theorem and its corollaries and Remark 1 may be regarded as generalizations of results of A. Tarski [7, (II)] and D. Scott [5]. Since both of these are concerned with Zermelo-Fraenkel set theory, the role of classes in this paper is played by formulae in [7] and [5]. In [5], reference is made to a similar idea of A. P. Morse. In [7, (III)] it is observed anew that the universe (which turns out to be $\mathscr{H}(0)=\cup\left\{\mathscr{F}_{\alpha} \alpha(0) \mid \alpha \in \mathcal{O}\right\}$ in [7]) is effectively covered by a well-ordered "sequence" of sets; this of course is clear in [8] and [1, VI, §16], but is done in |7] via an intrinsic theory of ranks. 
CoRollary 1.2. Suppose that each subclass of $\mathscr{U} \times \mathscr{P}\left(\mathscr{U}_{0}\right)$ is [perfectly] amenable, and suppose that the axiom of foundation holds. Then each class is [perfectly] amenable.

In the rest of this paper $\mathfrak{A}$ (resp., $\mathfrak{A}_{0}$ ) will be the statement that every class is amenable (resp., perfectly amenable).

REMARK 1. Consider the statements:

(i) $\mathscr{U}_{0}$ is void,

(ii) $\mathscr{U}_{0}$ is a set,

(iii) $\mathscr{U}_{0}$ is equi-potent to a subclass of $\mathscr{O}$,

(iv) There is a function $\tau$ from $\mathscr{O}$ to $\mathscr{P}\left(\mathscr{U}_{0}\right)$ such that $\mathscr{U}_{0}=$ $\mathrm{U} \operatorname{im}(\tau)$.

It is easily established that

$$
\text { (i) } \Rightarrow \text { (ii) } \Rightarrow \text { (iii) } \Rightarrow \text { (iv) . }
$$

If the axiom of foundation holds, it is easily established from Corollary 1.2 that

$$
\text { (iv) } \Rightarrow \mathfrak{U}_{0} \Rightarrow \mathfrak{U} \text {. }
$$

Theorem 2. Suppose $\mathfrak{A}$. Suppose that $\mathscr{C}$ is a class and that $\mathscr{R} \subset \mathscr{C} \times \mathscr{C}$ is a transitive and anti-reflexive relation. Suppose that every subset of $\mathscr{C}$ which is well-ordered by $\mathscr{R}$ has an $\mathscr{R}$-upper bound in $\mathscr{C}$. Then either $\mathscr{C}$ has an $\mathscr{R}$-maximal member or for each $\alpha \in \mathcal{O}$, $\mathscr{C}$ has a subset of type $\alpha$ under $\mathscr{R} \cdot{ }^{11,12}$

Proof. Let $\mathscr{W}$ be the class of all nonvoid subsets of $\mathscr{C}$ which are well-ordered by $\mathscr{R}$, and let $\mathscr{T} \subset \mathscr{W} \times \mathscr{C}$ be the relation such that for all $W \in \mathscr{W}$ and $c \in \mathscr{C}, W \mathscr{T} c$ if and only if $w \mathscr{R} c$ for each $w \in W$. By $\mathfrak{A}$ there is a function, say $\Gamma$, making $\mathscr{T}$ amenable.

Consider $c \in \mathscr{C}$ and $\alpha \in \mathcal{O}$ such that $\alpha>0$. Let $\Gamma_{\alpha}(c)$ be the class of all functions $f$ from $\alpha$ to $\mathscr{C}$ such that $f(0)=c$ and such that for each $\beta \in \alpha$ with $\beta>0, f(\beta) \in \Gamma(\operatorname{im}(f \mid \beta))$. Then $\Gamma_{\alpha}(c)$ is a set. To prove this consider $\gamma \in \mathcal{O} \backslash\{0\}$ such that $\Gamma_{\gamma}(c)$ is not a set while $\Gamma_{\beta}(c)$ is a set for each $\beta \in \gamma \backslash\{0\}$. If $\gamma=1$, then $\Gamma_{\gamma}(c)=\{\{[0, c]\}\}$ is a set contrary to assumption. If $\gamma>1$ is not a limit ordinal, then $\Gamma_{\gamma}(c)$ is equi-potent to a subclass of

$$
\Gamma_{\gamma-1}(c) \times \bigcup\left\{\Gamma(\operatorname{im}(f)) \mid f \in \Gamma_{\gamma-1}(c)\right\}
$$

${ }^{11}$ The set $L \subset \mathscr{C}$ has type $\alpha$ under $\mathscr{R}$ if and only if there is a one-to-one $\mathscr{R}$-increasing function from $\alpha$ onto $L$.

12 This generalized Zorn's lemma, under the assumption that the axiom of foundation and some condition such as (ii) (preferrably (i)) of Remark 1 hold, was suggested to the writer by the referee. An earlier version of this paper used transfinite recursion arguments rather than an analogue of Zorn's lemma. 
and hence is a set contrary to assumption. If $\gamma>1$ is a limit ordinal, then $\Gamma_{\gamma}(c)$ is equi-potent to a class of functions from $\gamma$ to

$$
\bigcup\left\{\Gamma_{\beta}(c) \mid 0<\beta<\gamma\right\}
$$

and hence is a set contrary to assumption. Thus there is no first $\gamma \in \mathcal{O}$, and hence no $\gamma \in \mathcal{O}$, such that $\Gamma_{\gamma}(c)$ is not a set; thus $\Gamma_{\alpha}(c)$ is a set. Let

$$
A_{\alpha}(c)=\bigcup\left\{\Gamma_{\beta}(c) \mid 0<\beta \leqq \alpha\right\} .
$$

By Zorn's lemma $A_{\alpha}(c)$ has a member $f$ maximal in $\Lambda_{\alpha}(c)$ under setinclusion. If $\operatorname{dom}(f)=\alpha$, then $\operatorname{im}(f)$ is a subset of $\mathscr{C}$ of type $\alpha$ under $\mathscr{R}$. Suppose $\operatorname{dom}(f) \neq \alpha$. Then $\operatorname{dom}(f)$ is a nonlimit ordinal, for otherwise $\operatorname{im}(f)$ has an $\mathscr{R}$-upper bound $d$, which may be assumed to be a member of $\Gamma(\operatorname{im}(f))$ and thus results in

$$
f \bigcup\{[\operatorname{dom}(f), d]\} \in \Lambda_{\alpha}(c)
$$

contrary to the maximality of $f$ in $A_{\alpha}(c)$. Since $\operatorname{dom}(f)$ is a nonlimit ordinal $<\alpha$ and $f$ is maximal in $\Lambda_{\alpha}(c)$, it may be verified that $f(\operatorname{dom}(f)-1)$ is $\mathscr{R}$-maximal.

The conclusion of the theorem follows from the preceding paragraph if $\mathscr{C} \neq 0$. Now $0 \subset \mathscr{C}$ is well-ordered by $\mathscr{R}$ and has an $\mathscr{R}$-upper bound in $\mathscr{C}$. Hence $\mathscr{C} \neq 0$. Q.e.d.

REMARK 2. Theorem 2 remains valid if instead of supposing $\mathfrak{A}$ one supposes that $\mathscr{C} \subset \mathscr{V}$ for some subuniverse $\mathscr{V}$ each subclass of which is amenable. (Cf. the second and third paragraphs of $\S 3$.)

5. The predicate $\mathfrak{Q}$ and its completion theorem. $B y \mathfrak{A}(\mathscr{A}, \prec$; $\left.\pi, \rho, \delta, \pi^{\prime}\right)$ will be meant the conjunction of (1)-(6) below.

(1) $\mathscr{A}$ is a class, and $\prec \subset \mathscr{A} \times \mathscr{A}$ is reflexive on $\mathscr{A}$ and partially orders A $^{13}$

(2) For each set $L \subset \mathscr{A}$ well-ordered by $\prec$ there is at least one $b \in \mathscr{A}$ that $a \prec b$ for each $a \in L$.

(3) $\pi$ is a function from $\mathscr{A}$ to the class of all sets.

(4) $\rho$ is a function on $\prec$ such that (4a)-(4c) below hold for all $a, b$, $c \in \mathscr{A}$, where we write " $\rho_{a}^{b "}$ instead of " $\rho([a, b])$ ".

(4a) If $a \prec b$, then $\rho_{a}^{b}$ is a function from $\pi(a)$ to $\pi(b)$.

(4b) $\rho_{a}^{a}$ is the identity function on $\pi(a)$.

(4c) If $a<b$ and $b<c, \rho_{a}^{c}=\rho_{b}^{c} \circ \rho_{a}^{b}$.

(5) $\delta$ is a nonzero limit ordinal number. For each set $L \subset \mathscr{A}$ of type $\delta$ under $\prec, L$ has an $\prec$-upper bound $b \in \mathscr{A}$ such that $\pi(b)=$

${ }_{13} \prec$ partially orders $\mathscr{A}$ if and only if $\mathscr{A}$ is a class, $\prec$ is a relation, $\prec$ is transitive on $\mathscr{A}$, and for all $a, b \in \mathscr{A}$, if $a \prec b$ and $b \prec a$, then $a=b$. 


\section{$\bigcup\left\{\operatorname{im}\left(\rho_{a}^{b}\right) \mid a \in L\right\}$.}

(6) $\pi^{\prime}$ is a function on $\mathscr{A}$ such that $\pi^{\prime}(a)$ is a subset of $\pi(a)$ for each $a \in \mathscr{A}$ and such that for all $a, b \in \mathscr{A}$ with $a \prec b, \rho_{a}^{b}(p) \in \pi^{\prime}(b)$ for each $p \in \pi^{\prime}(a)$.

Examples of $\mathfrak{Q}$ will be given later; at this point $\S 1$ should be kept in mind for motivation. Similar predicates will be considered later.

Suppose $Q\left(\mathscr{A}, \prec ; \pi, \rho, \delta, \pi^{\prime}\right)$. (Then $\mathscr{A}$ is determined by any one of $\prec, \pi, \rho, \pi^{\prime}$ since $\mathscr{A}$ is the domain of each of $\prec, \pi, \pi^{\prime}$, and $\operatorname{dom}(\rho)$.) For each element $a, a$ will be called $\prec-\pi-\rho-\pi^{\prime}$-complete if and only if $a \in \mathscr{A}$ and for each $p \in \pi(a)$ and each $b \in \mathscr{A}$ with $a \prec b, \quad \rho_{a}^{b}(p) \in \pi^{\prime}(b)$ only if $p \in \pi^{\prime}(a)$.

The following theorem is the first completion theorem in this paper which can be applied to the type of situation described in $\S 1$.

Theorem 3. Suppose $\mathfrak{A}$. Suppose $Q\left(\mathscr{A}, \prec ; \pi, \rho, \pi^{\prime}\right)$. Then for each $a \in \mathscr{A}$ there is some $\prec-\pi-\rho-\pi^{\prime}$-complete $b$ such that $a \prec b$.

Proof. Consider $a \in \mathscr{A}$. Let $\mathscr{C}_{a}=\{b \mid a \prec b\}$. There in a relation $\mathscr{R}_{a} \subset \mathscr{C}_{a} \times \mathscr{C}_{a}$ such that for all $b, c \in \mathscr{C}_{a}, b \mathscr{R}_{a} c$ if and only if $b \prec c$ and there is $p \in \pi(a)$ such that $\rho_{a}^{b}(p) \notin \pi^{\prime}(b)$ and $\rho_{a}^{c}(p) \in \pi^{\prime}(c)$. It may be verified that for all $b, c, d \in \mathscr{C}_{a}$, if both $b \prec c$ and $c \mathscr{R}_{a} d$ or both $b \mathscr{R}_{a} c$ and $c \prec d$, then $b \mathscr{R}_{a} d$. Then further, $\mathscr{R}_{a}$ is transitive and anti-reflexive. Consider any set $L \subset \mathscr{C}_{a}$ well-ordered by $\mathscr{R}_{a}$. If $L=0, a$ is an $\mathscr{R}_{a^{-}}$ upper bound of $L$. If $L$ has an $\mathscr{R}_{a}$-maximum, $L$ has an $\mathscr{R}_{a}$-upper bound. If $L \neq 0$ and $L$ has no $\mathscr{R}_{a}$-maximum, then, by (2), $L$ has an $\prec$-upper bound $d$, and it may be verified that $d \in \mathscr{C}_{a}$ and that $d$ is an $\mathscr{R}_{a}$-upper bound of $L$. Let $\alpha$ be the smallest infinite cardinal greater than the cardinal of $\pi(a)$. Suppose $L \subset \mathscr{C}_{a}$ has type $\alpha$ under $\mathscr{R}_{a}$. There is a one-to-one $\mathscr{R}_{a}$-increasing function $\varphi$ from $\alpha$ onto $L$. By the axiom of choice there is a function $\psi$ from $\alpha$ into $\pi(a)$ such that for each $\lambda \in \alpha, \quad \rho_{a}^{\varphi(\lambda)}(\psi(\lambda)) \notin \pi^{\prime}(\varphi(\lambda))$ and $\rho_{a}^{\varphi(\lambda+1)}(\psi(\lambda)) \in \pi^{\prime}(\varphi(\lambda+1))$. It may be verified that $\psi$ is one-to-one. Hence $\pi(a)$ has cardinal $\geqq \alpha$, contradiction. Thus no subset of $\mathscr{C}_{a}$ has type $\alpha$ under $\mathscr{R}_{a}$. By Theorem 2, $\mathscr{C}_{a}$ has an $\mathscr{R}_{a}$-maximal member.

Again consider $a \in \mathscr{A}$. There is a relation $\mathscr{S}_{a} \subset \mathscr{C}_{a} \times \mathscr{C}_{a}$ such that for all $b, c \in \mathscr{C}_{a}, b \mathscr{S}_{a} c$ if and only if $b \neq c$ and $c$ is an $\mathscr{R}_{b}$-maximal member of $\mathscr{C}_{b}$. Thus $b \mathscr{S}_{a} c$ implies $a \prec b \prec c$. It may be verified that for all $b, c, d \in \mathscr{C}_{a}$, if both $b \prec c$ and $c \mathscr{S}_{a} d$ or both $b \mathscr{S}_{a} c$ and $c \prec d$, then $b \mathscr{S}_{a} d$. Then further, $\mathscr{S}_{a}$ is transitive and anti-reflexive. Consider any set $L \subset \mathscr{C}_{a}$ well-ordered by $\mathscr{S}_{a}$. If $L=0, a$ is an $\mathscr{S}_{a}$-upper bound of $L$. If $L$ has an $\mathscr{S}_{a}$-maximum, $L$ has an $\mathscr{S}_{a}$-upper bound. If $L \neq 0$ and $L$ has no $\mathscr{S}_{a}$-maximum, then, by (2), $L$ has an $\prec$-upper bound $d$, and it may be verified that $d \in \mathscr{C}_{a}$ is an $\mathscr{S}_{a}$-upper bound of $L$. 
Suppose $L \subset \mathscr{C}_{a}$ has type $\delta$ under $\mathscr{S}_{a}$. By (5), $L$ has an $\prec$-upper bound $d$ such that $\pi(d)=\bigcup\left\{\operatorname{im}\left(\rho_{b}^{d}\right) \mid b \in L\right\}$. Consider a such $d$. Then $a \prec d$. It is desired to prove that $d$ is $\prec-\pi-\rho-\pi^{\prime}$-complete (briefly, complete). Consider $q \in \pi(d)$ and $e \in \mathscr{A}$ such that $e \neq d, d \prec e$, and $\rho_{d}^{e}(q) \in \pi^{\prime}(e)$. For some $b \in L$ and $p \in \pi(b), q=\rho_{b}^{d}(p)$. Consider such $b$ and $p$. Now b $\mathscr{S}_{a} d$. Hence $d$ is $\mathscr{R}_{b}$-maximal, and not $d \mathscr{R}_{b} e$. Since $\rho_{b}^{e}(p)=\rho_{a}^{e}(q) \in \pi^{\prime}(e)$ and not $d \mathscr{R}_{b} e, q=\rho_{b}^{d}(p) \notin \pi^{\prime}(d)$ is impossible. Hence $q \in \pi^{\prime}(d)$. Thus $d$ is complete.

Suppose there is no set $L \subset \mathscr{C}_{a}$ having type $\delta$ under $\mathscr{S}_{a}$. By Theorem $2, \mathscr{C}_{a}$ has an $\mathscr{S}_{a}$-maximal member, say $b$. Then $a \prec b$. By the first paragraph of the proof, $\mathscr{C}_{b}$ has an $\mathscr{R}_{b}$-maximal member, say $c$. If $b \neq c$, then $b \mathscr{S}_{a} c$, and the $\mathscr{S}_{a}$-maximality of $b$ is contradicted. Hence $b=c$. Thus $b$ is $\mathscr{R}_{b}$-maximal. Thus $b$ is complete.

Thus $a \prec b$ for some complete $b$ in any event. Q.e.d.

Remark 3. Suppose 21. Suppose (1)-(4) and (6) are assumed (thus (5) is not assumed). Then for each $a \in \mathscr{A}$ there is $b \in \mathscr{A}$ such that $a<b$ and such that for each $p \in \pi(a)$ and each $c \in \mathscr{A}$ with $b \prec c, \rho_{a}^{c}(p) \in \pi^{\prime}(c)$ only if $\rho_{a}^{b}(p) \in \pi^{\prime}(b)$. This follows from the fact that the first paragraph of the proof of Theorem 3 does not use (5).

Remark 4. Theorem 3 and Remark 3 remain valid if instead of supposing $\mathfrak{A}$ one supposes that $\mathscr{A} \cup \pi \subset \mathscr{V}$ for some subuniverse $\mathscr{V}$ each subclass of which is amenable. (Cf. the second and third paragraphs of $\S 3$.)

By $\mathfrak{I}^{*}\left(\mathscr{A}, \prec ; \pi, \delta, \pi^{\prime}\right)$ will be meant that $\mathfrak{Q}\left(\mathscr{A}, \prec ; \pi, \rho, \delta, \pi^{\prime}\right)$ holds where $\rho([a, b])=\{[p, p] \mid p \in \pi(a)\}$ for all $a, b \in \mathscr{A}$ with $a \prec b$. Consider $\mathscr{A}, \prec, \pi, \rho, \delta, \pi^{\prime}$ so given. (4) may be restated: for all $a, b \in \mathscr{A}$ with $a \prec b, \pi(a) \subset \pi(b)$. (5) and (6) may be similarly simplified.

Suppose $\mathfrak{\Omega}^{*}\left(\mathscr{A}, \prec ; \pi, \delta, \pi^{\prime}\right)$. For each element $a, a$ will be called $\prec-\pi-\pi^{\prime}$-complete if and only if $a$ is $\prec-\pi-\rho-\pi^{\prime}$-complete where $\rho$ is given as in the preceding paragraph. Then for each $a \in \mathscr{A}, a$ is $\prec-\pi-\pi^{\prime}$-complete if and only if for each $b \in \mathscr{A}$ with $a \prec b, \pi^{\prime}(a)=\pi(a) \cap \pi^{\prime}(b){ }^{14}$

6. The predicates $\mathfrak{P}$ and $\Re$. $B y \mathfrak{P}(\mathscr{A}, \mathscr{B}, \Psi, \prec)$ will be meant the conjunction of (7)-(11) below and (2) above.

(7) $\mathscr{A}$ is a class, and $\mathscr{B}$ is a function from $\mathscr{A}$ to the class of all sets.

(8) $\Psi$ is a class of triples $(f, a, b)$ such that $a, b \in \mathcal{A}$ and $f$ is $a$ function from $\mathscr{B}(a)$ into $\mathscr{B}(b) .{ }^{15}$

14 This elegant formulation of $\prec-\pi-\pi^{\prime}$-completeness is due to the referee.

15 In the rest of this paper certain terminology is as follows. For each set $X$ an $X$ tuple is a function on $X$. A triple is a 3-tuple. A couple is a 2-tuple $(x, p)=\{[0, x],[1, y]\}$ and is to be distinguished from the pair $[x, y]$. 
(9) If $(f, a, b),(g, b, c) \in \Psi$, then $(g \circ f, a, c) \in \Psi$.

(10) If $a \in \mathscr{A}, X$ is a set, and $f$ is a one-to-one function from $\mathscr{B}(a)$ onto $X$, then there is one and only one $b \in \mathscr{A}$ such that $\mathscr{B}(b)=$ $X$ and $(f, a, b),\left(f^{-1}, b, a\right) \in \Psi$.

(11) $\prec \subset \mathscr{A} \times \mathscr{A}$. For all $a, b \in \mathscr{A}, a \prec b$ if and only if $\left(f_{a}, a, b\right) \in \Psi$ where $f_{a}=\{[x, x] \mid x \in \mathscr{B}(a)\}$.

The statement $\mathfrak{P}(\mathscr{A}, \mathscr{B}, \Psi, \prec)$ will often hold in a situation in which $\mathscr{A}$ is a species of mathematical systems, $\mathscr{B}(a)$ is the base set of $a$ for each $a \in \mathscr{A}, f$ is a monomorphism from a to $b$ for each $(f, a, b) \in \Psi$, and $\prec$ is the subsystem relation for $\mathscr{A}$. In this same situation many familiar examples of $\mathscr{A}$ and $\prec$ are such that if $L$ is given as in (2), $L$ has a least upper bound under $\prec$; this is the case in Examples 1 and 2 following but is not the case in Example 3 .

Example 1. Let $\mathscr{A}$ be the class of all fields (i.e., triples $(F,+, \cdot)$ with the usual properties), and let $\prec$ be the subfield relation. Let

$$
\begin{gathered}
\mathscr{B}=\{[(F,+, \cdot), F] \mid(F,+, \cdot) \in \mathscr{A}\}, \\
\Psi=\{(f, a, b) \mid a, b \in \mathscr{A} ; f \text { is a monomorphism from } a \text { to } b\} .
\end{gathered}
$$

Then $\mathfrak{P}(\mathscr{A}, \mathscr{B}, \Psi, \prec)$.

ExAmple 2. Let $\mathscr{A}$ be the class of all groups (i.e., couples $(G, \cdot)$ with the usual properties), and let $\prec, \mathscr{B}, \Psi$ be defined in the obvious way in analogy with Example 1. Then $\mathfrak{P}(\mathscr{A}, \mathscr{B}, \Psi, \prec)$.

ExAmple 3. Let $\mathscr{A}$ be the class of all topological spaces (i.e., couples $(X, T)$ with the usual properties, $T$ consisting of the open subsets of $X$ ), and let $\prec$ be the topological subspace relation. Let

$$
\begin{aligned}
\mathscr{B} & =\{[(X, T), X] \mid(X, T) \in \mathscr{A}\}, \\
\Psi & =\{(f, a, b) \mid a, b \in \mathscr{A} ; f \text { is a homeomorphism of } a \text { into } b\} .
\end{aligned}
$$

Then $\mathfrak{P}(\mathscr{A}, \mathscr{B}, \Psi, \prec)$. Here (2) may be proved as follows. If $L \subset \mathscr{A}$ is a set well-ordered by $\prec$, let $\sigma(L)=(Y, U)$ where $Y=\bigcup\{\mathscr{B}(\alpha) \mid a \in L\}$ and $U=\{Z \mid Z \subset Y ; X \cap Z \in T$ for each $(X, T) \in L\}$. Given $L$ as just specified, $a \prec \sigma(L)$ for each $a \in L$, but not necessarily is $\sigma(L)$ a $\prec$-supremum of $L$.

Lemma 1. Suppose $\mathfrak{P}(\mathscr{A}, \mathscr{B}, \Psi, \prec)$, or suppose merely (7)-(11). Then $\prec$ is reflexive on $\mathscr{A}$ and partially orders $\mathscr{A}$.

Proof. Consider $a \in \mathscr{A}$. By (10) there is $b \in \mathscr{A}$ such that $\mathscr{B}(b)=$ $\mathscr{B}(a)$ and $\left(f_{a}, a, b\right),\left(f_{a}^{-1}, b, a\right) \in \Psi$, where $f_{a}=f_{a}^{-1}=f_{a} \circ f_{a}$ is given as in (11), and $\left(f_{a}, a, a\right) \in \Psi$ by (9). Hence $a \prec a$ by (11). Thus $\prec$ is 
reflexive on $\mathscr{A}$. By (7), (8), (11), and (9), $\prec$ is transitive. If $c, d \in \mathscr{A}$ are such that $c \prec d$ and $d \prec c$, then $\left(f_{c}, c, d\right),\left(f_{d}, d, c\right) \in \Psi$ by $(11), \mathscr{B}(c)=$ $\mathscr{S}(d)$ by (8), and $c=d$ by (10) (for $f_{a}=f_{c}^{-1}$, and $\left(f_{c}, c, c\right),\left(f_{c}^{-1}, c, c\right) \in \Psi$ by $\prec$ being reflexive on $\mathscr{A})$. Thus $\prec$ partially orders $\mathscr{A}$. Q.e.d.

Lemma 2. Suppose $\mathfrak{B}(\mathscr{A}, \mathscr{B}, \Psi, \prec)$, or suppose merely (7)-(11). If $c \prec a$, then $\mathscr{B}(c) \subset \mathscr{B}(a)$. Consider $(f, a, b) \in \Psi$ such that $\left(f^{-1}, b, a\right) \in \Psi$ (one could say that $f$ is an isomorphism from a to $b$ ). Let $\mathscr{F}$ be the class of all pairs $[c, d]$ such that $c \prec a, d \prec b,(f \mid \mathscr{P}(c), c, d) \in \Psi$, and $\left(f^{-1} \mid \mathscr{B}(d), d, c\right) \in \Psi$. Then $\mathscr{F}$ is a one-to-one function from $\{c \mid c \prec a\}$ onto $\{d \mid d \prec b\}$; moreover, for all $c_{1} \prec a$ and $c_{2} \prec a, c_{1} \prec c_{2}$ if and only if $\mathscr{F}\left(c_{1}\right) \prec \mathscr{F}\left(c_{2}\right)$. (One could say that $\mathscr{F}$ is a $\prec$-preserving one-toone correspondence between the class of all subsystems of a and the class of all subsystems of $b$ induced by $f$.)

Proof. If $c \prec a$, then $\mathscr{B}(c) \subset \mathscr{B}(a)$ by (7), (8), and (11).

For the rest of Lemma 2 consider $f, a, b, \mathscr{F}$ as specified.

Consider $c \prec a$. Then $\mathscr{B}(c) \subset \mathscr{B}(a)$. By (10) there is a unique $d \in \mathscr{A}$ with $(f \mid \mathscr{B}(c), c, d),\left((f \mid \mathscr{B}(c))^{-1}, d c\right) \in \Psi$. Then also, where $f_{c}$ and $f_{a}$ are defined as in (11),

$$
\left(f_{a}, d, b\right)=\left(f \circ f_{c} \circ(f \mid \mathscr{P}(c))^{-1}, d, b\right) \in \Psi
$$

by (11) and two applications of (9). Hence $d \prec b$. Moreover, $(f \mid \mathscr{B}(c))^{-1}=$ $f^{-1} \mid \mathscr{B}(d)$ since $\left((f \mid \mathscr{B}(c))^{-1}, d, c\right) \in \Psi$. It follows that $[c, d] \in \mathscr{F}$. Consider also $e \in \mathscr{A}$ such that $[c, e] \in \mathscr{F}$. By $(8), \mathscr{B}(e)=\operatorname{dom}\left(f^{-1} \mid \mathscr{B}(d)\right)=$ $\mathscr{B}(d)$ since $f$ is one-to-one $\left(f^{-1}\right.$ is a function by (8)), and $d=e$ by (10).

It follows that $\mathscr{F}$ is a function from $\{c \mid c<a\}$ into $\{d \mid d<b\}$. Symmetrically, $\mathscr{F}^{-1}$ is a function from $\{d \mid d \prec b\}$ into $\{c \mid c \prec a\}$. Hence $\mathscr{F}$ is a one-to-one function from $\{c \mid c \prec a\}$ onto $\{d \mid d \prec b\}$.

Now consider $c_{1}, c_{2} \in \mathscr{A}$ such that $c_{1} \prec c_{2} \prec a$. Then $\left(f \mid \mathscr{B}\left(c_{2}\right), c_{2}, \mathscr{F}\left(c_{2}\right)\right)$, $\left(\left(f \mid \mathscr{B}\left(c_{2}\right)\right)^{-1}, \mathscr{F}\left(c_{2}\right), c_{2}\right) \in \Psi$. Let $\mathscr{G}$ be defined in terms of $f \mid \mathscr{B}\left(c_{2}\right), c_{2}$, $\mathscr{F}\left(c_{2}\right)$ as $\mathscr{F}$ is defined in terms of $f, a, b$. Then $\mathscr{G} \subset \mathscr{F}$. Moreover, $\mathscr{F}\left(c_{1}\right) \prec \mathscr{F}\left(c_{2}\right)$ since $\mathscr{G}$ is a function from $\left\{c \mid c \prec c_{2}\right\}$ onto $\left\{d \mid d \prec \mathscr{F}\left(c_{2}\right)\right\}$. Hence $\mathscr{F}\left(c_{1}\right)=\mathscr{G}\left(c_{1}\right) \prec \mathscr{F}\left(c_{2}\right)$.

Symmetrically, if $c_{1} \prec a, c_{2} \prec a$, and $\mathscr{F}\left(c_{1}\right) \prec \mathscr{F}\left(c_{2}\right)$, then $c_{1}=$ $\mathscr{F}^{-1}\left(\mathscr{F}\left(c_{1}\right)\right) \prec \mathscr{F}^{-1}\left(\mathscr{F}\left(c_{2}\right)\right)=c_{2}$. Q.e.d.

By $\Re\left(\mathscr{A}, \mathscr{S}, \Psi, \prec ; \pi, \rho, \delta, \pi^{\prime} ; S, \theta\right)$ will be meant the conjunction of (1)-(11) above and (12)-(13) below, i.e., the conjunction of $\mathfrak{P}(\mathscr{A}, \mathscr{B}, \Psi, \prec), \mathfrak{Q}\left(\mathscr{A}, \prec ; \pi, \rho, \delta, \pi^{\prime}\right),(12)$, and (13); by Lemma 1 , (1) is redundant.

(12) $S$ is a set; for each $a \in \mathscr{A}, \pi(a) \in \mathscr{H}(\{a\} \cup S)=\mathscr{H}(\mathscr{B}(a) \cup S)$.

(13) $\theta$ is a function on $\left\{(f, a, b) \mid(f, a, b),\left(f^{-1}, b, a\right) \in \Psi\right\}$, and (13a)-(13c) below hold, where we write " $(\theta f)_{a}^{b "}$ " instead of " $\theta((f, a, b))$ ". 
(13a) If $(f, a, b) \in \operatorname{dom}(\theta)$, then $(\theta f)_{a}^{b}$ is a function from $\pi(a)$ into $\pi(b)$, and $(\theta f)_{a}^{b}(p) \in \pi^{\prime}(b)$ for each $p \in \pi^{\prime}(a)$.

(13b) If $a \in \mathscr{A}$ and $f_{a}$ is the identity function on $\mathscr{B}(a)(c f .(11))$, then $\left(\theta f_{a}\right)_{a}^{a}$ is the identity function on $\pi(a)$.

(13c) If $(f, a, b) \in \operatorname{dom}(\theta), \quad c \prec a, \quad d \prec b, \quad g=f \mid \mathscr{B}(a)$, and $(g, c, d) \in \operatorname{dom}(\theta)$, then $\rho_{d}^{b} \circ(\theta g)_{c}^{d}=(\theta f)_{a}^{b} \circ \rho_{c}^{a} . \quad(C f$. Lemma 2.)

Both $\cong$ and $\Re$ furnish settings for problems of the type described in $\S 1$, but $\Re$ entails a functorial type of structure ignored by $\Omega$ and present in most explicit problems of the type described in $\S 1$.

By $\Re^{*}\left(\mathscr{A}, \mathscr{B}, \Psi, \prec ; \pi, \delta, \pi^{\prime} ; S, \theta\right)$ will be meant that $\Re(\mathscr{A}, \mathscr{R}, \Psi, \prec$; $\left.\pi, \rho, \delta, \pi^{\prime} ; S, \theta\right)$ holds where $\rho([a, b])=\{[p, p] \mid p \in \pi(a)\}$ for all $a, b \in \mathscr{A}$ with $a \prec b$.

EXAmple 1 (continued). Let $\pi$ and $\pi^{\prime}$ be functions on $\mathscr{A}$ such that for each $a=(\mathscr{F},+, \cdot) \in \mathscr{A}, \pi(\alpha)$ is the set of all finite sequences ${ }^{16}$ of members of $\mathscr{F}$ and

$$
\pi^{\prime}(a)=\left\{p \in \pi(a) \mid \sum_{j \in \operatorname{dom}(p)} p(j) x^{j}=0 \quad \text { for some } \quad x \in F\right\} .
$$

Let $\theta$ be the function with domain indicated in (12) such that (in the notation of (12)) for each $(f, a, b) \in \operatorname{dom}(\theta),(\theta f)_{a}^{b}$ is a function from $\pi(a)$ into $\pi(b)$, and $(\theta f)_{a}^{b}=f \circ p$ for each $p \in \pi(a)$. Then $\Re^{*}(\mathscr{A}, \mathscr{B}, \Psi, \prec$; $\left.\pi, \omega, \pi^{\prime} ; 0, \theta\right){ }^{17} \quad$ A field $a \in \mathscr{A}$ is $\prec-\pi-\pi^{\prime}$-complete if and only if $a$ is algebraically closed (algebraically complete would be a better term). ${ }^{18}$

EXAmple 2 (continued). Let $\mu \in \mathcal{O}$. Let $\pi$ be the function on $\mathscr{A}$ such that for each $a=(G, \cdot) \in \mathscr{A}, \pi(a)$ is the set of all couples $(X, Y)$ such that (i) and (ii) below hold.

(i) $X \cup Y$ is a set of triples $(g, h, j)$ of finite sequences $g, h, j$ such that $\operatorname{dom}(g)=\operatorname{dom}(h)=\operatorname{dom}(j), \operatorname{im}(g) \subset G, \operatorname{im}(h)$ is a set of integers, and $\operatorname{im}(j) \subset \boldsymbol{\aleph}_{\mu}$.

(ii) The cardinal of $X \cup Y$ is $\left\langle\boldsymbol{\gamma}_{\mu}\right.$.

Let $\pi^{\prime}$ be the function on $\mathscr{A}$ such that for each $a=(G, \cdot) \in \mathscr{A}, \pi^{\prime}(a)$ is the set of all $(X, Y) \in \pi(a)$ for which there is a function $k: \aleph_{\mu} \rightarrow G$ such that ( $e$ being the neutral element of $G$ under $\cdot$ )

$$
\prod_{\alpha \in \operatorname{dum}(f)}\left[g(\alpha) k(j(\alpha))^{h(\alpha)}\right]\left\{\begin{array}{l}
=e \text { for each }(g, h, j) \in X, \\
\neq e \text { for each }(g, h, j) \in Y .
\end{array}\right.
$$

${ }_{16}$ A finite sequence is any element which is an $n$-tuple for some $n \in \omega$.

$17 \pi(a)$ will serve as a set-theoretically meaningful substitute for the "class" of polynomial equations over $a$. $\pi^{\prime}(a)$ gives rise to the "class" of polynomial equations over $a$ with solutions in $a$.

${ }_{18}$ As is well-known, a field $a$ is algebraically closed if and only if every nontrivial polynomial equation over $a$ has a root in $a$. The proof of this of course uses information about the nature of the elements of $\mathscr{A}$; information of this type has no bearing on the methods of this paper. 
Let $\theta$ be the function with domain indicated in (12) such that (in the notation of (12)) for each $(f, a, b) \in \operatorname{dom}(\theta),(\theta f)_{a}^{b}$ is a function from $\pi(a)$ into $\pi(b)$, and for each $(X, Y) \in \pi(a)$,

$$
(\theta f)_{a}^{b}((X, Y))=(\{(f \circ g, h, j) \mid(g, h, j) \in X\},\{(f \circ g, h, j) \mid(g, h, j) \in Y\}) .
$$

Then $\mathfrak{R}^{*}\left(\mathscr{A}, \mathscr{B}, \Psi, \prec ; \pi, \aleph_{\mu+1}, \pi^{\prime} ; 0, \theta\right) .^{19} \quad$ A group $a \in \mathscr{A}$ is $\prec-\pi-\pi^{\prime}-$ complete if and only if $a$ is algebraically closed $\left(\boldsymbol{\gamma}_{\mu}\right)$ in the sense of W. R. Scott [6] (algebraically complete $\left(\mathbf{S}_{\mu}\right)$ would be a better term).

7. The completion theorem for $\Re$. The second completion theorem in this paper is Theorem 4 below and can be applied to the type of situation described in $\S 1$ without the assumption $\mathfrak{A}$.

Lemma 3. Suppose $\mathfrak{P}(\mathscr{A}, \mathscr{B}, \Psi, \prec)$; suppose $\mathscr{Y}$ is a subuniverse, and suppose that for each $a \in \mathscr{A}, a \in \mathscr{Y}$ if and only if $\mathscr{B}(a) \in \mathscr{V}$. Then $\mathfrak{P}(\mathscr{A} \cap \mathscr{Y}, \mathscr{B} \cap \mathscr{Y}, \Psi \cap \mathscr{Y}, \prec \cap \mathscr{Y})$.

Proof. The proofs of the statements obtained from (7)-(11) by replacing $\mathscr{A}, \mathscr{B}, \Psi, \prec$ by $\mathscr{A} \cap \mathscr{Y}^{-}, \mathscr{B} \cap \mathscr{V}^{\wedge}, \Psi \cap \mathscr{Y}, \prec \cap \mathscr{Y}$ respectively are routine.

Consider any set $L \subset \mathscr{A} \cap \mathscr{V}$ well-ordered by $\prec \cap \mathscr{Y}$. By (2), $L$ has an 〈-upper bound, say $b$. Let $B_{L}=\bigcup\{\mathscr{B}(a) \mid a \in L\}$. By Lemma 2, $B_{L} \subset \mathscr{B}(b)$. There is some $\alpha \in \mathscr{O}$ such that $\alpha \backslash B_{L}$ is equi-potent with $\mathscr{B}(b) \backslash B_{L}$, and for such an $\alpha$ there is a one-to-one function $f$ from $\mathscr{B}(b)$ onto $\alpha \cup B_{L}$ such that $f(x)=x$ for each $x \in B_{L}$. Consider such $\alpha$ and $f$. By (10) there is $c \in \mathscr{A}$ such that $\mathscr{B}(c)=\alpha \cup B_{L}$ and $(f, b, c)$, $\left(f^{-1}, c, b\right) \in \Psi$; consider $a$ such $c$. For each $a \in \mathscr{A}$ let $f_{a}$ be as in (11). For each $a \in L,\left(f_{a}, a, b\right) \in \Psi$ by (11), hence (since also $(f, b, c) \in \Psi$ and $\left.f \circ f_{a}=f_{a}\right) \quad\left(f_{a}, a, c\right) \in T$ by (9), and hence $a \prec c$ by (11). Moreover, $\mathscr{B}(c)=\alpha \cup B_{L} \in \mathscr{Y}$, and hence $c \in \mathscr{Y}$.

The statement obtained from (2) by replacing $\mathscr{A}, \mathscr{B}, \Psi, \prec$ by $\mathscr{A} \cap \mathscr{Y}, \mathscr{B} \cap \mathscr{Y}, \Psi \cap \mathscr{V}, \prec \cup \mathscr{V}$ respectively follows from the previous paragraph. Q.e.d.

Lemma 4. Suppose $\Re\left(\mathscr{A}, \mathscr{B}, \Psi, \prec ; \pi, \rho, \delta, \pi^{\prime} ; S, \theta\right)$, and suppose $\mathscr{Y}$ is a subuniverse and $S \in \mathscr{Y}$. Then $\Re(\mathscr{A} \cap \mathscr{V}, \mathscr{B} \cap \mathscr{Y}, \Psi \cap \mathscr{Y}$, $\left.\prec \cap \mathscr{V} ; \pi \cap \mathscr{V}, \rho \cap \mathscr{V}, \delta, \pi^{\prime} \cap \mathscr{V} ; S, \theta \cap \mathscr{V}\right)$.

Proof. The obvious analogues of (2)-(13) must be proved (recall

${ }^{19} \pi(a)$ will serve as a set-theoretically meaningful substitute for the "class" of all systems of fewer than $\boldsymbol{\aleph}_{\mu}$ simultaneous equations and inequations over $a$ in fewer than $\boldsymbol{\aleph}_{\mu}$ unknowns, each equation (resp., inequation) equating (resp., inequating) a product of elements of $a$ and unknowns to the neutral element of $a . \pi^{\prime}(a)$ gives rise to the class of such systems with solutions in $a$. 
that (1) follows from (2)-(13)). By (12), for each $a \in \mathscr{A}, a \in \mathscr{Y}$ if and only if $\mathscr{B}(a) \in \mathscr{Y}$. Then for (2) and (7)-(11) apply Lemma 3. The proof of the analogue of the conjunction of (3) and (12) is routine. Then also the proofs of the analogues of (4), (6), and (13) are routine.

Consider any set $L \subset \mathscr{A} \cap \mathscr{V}$ of type $\delta$ under $\prec \cap \mathscr{Y}$. Let $b$ be given by (5). There are $B_{L}, \alpha$, and $c$ as in the second paragraph of the proof of Lemma 3. Then $c \in \mathscr{A} \cap \mathscr{V}$, and $a \prec c$ for each $a \in L$. Moreover, $(f, b, c),\left(f^{-1}, c, b\right) \in \Psi$, and hence $\pi(c)=\operatorname{im}\left((\theta f)_{b}^{c}\right)$ by (13). Let $\mathscr{F}$ be the class of all pairs $[d, e]$ such that $d \prec b, e \prec c,(f \mid \mathscr{B}(d), d, e) \in \Psi$, and $\left(f^{-1} \mid \mathscr{B}(e), e, d\right) \in \Psi$. By Lemma $2, \mathscr{F}$ is a one-to-one $\prec$-preserving function from $\{d \mid d \prec b\}$ onto $\{e \mid e \prec c\}$. Moreover, for each $a \in L$, $f \mid \mathscr{B}(a)=f_{a}$ where $f_{a}$ is as in (11), and hence $\mathscr{F}(a)=a$. By (5) and (13),

$$
\begin{aligned}
\pi(c) & =\operatorname{im}\left((\theta f)_{b}^{c}\right)=\bigcup\left\{\operatorname{im}\left((\theta f)_{b}^{c} \circ \rho_{a}^{b}\right) \mid a \in L\right\} \\
& =\bigcup\left\{\operatorname{im}\left(\rho_{a}^{c} \circ\left(\theta f_{a}\right)_{a}^{a}\right) \mid a \in L\right\}=\bigcup\left\{\operatorname{im}\left(\rho_{a}^{c}\right) \mid a \in L\right\} .
\end{aligned}
$$

The obvious analogue of (5) follows from the previous paragraph. Q.e.d.

Lemma 5. Suppose $\Re\left(\mathscr{A}, \mathscr{B}, \Psi, \prec ; \pi, \rho, \delta, \pi^{\prime} ; S, \theta\right)$, suppose $\mathscr{V}^{\wedge}$ is $a$ subuniverse, and suppose $S \in \mathscr{Y}$ and $a \in \mathscr{A} \cap \mathscr{V}$. Then $a$ is $\prec-\pi-\rho-\pi^{\prime}-$ complete if and only if $a$ is $(\prec \cap \mathscr{V})-(\pi \cap \mathscr{V})-(\rho \cap \mathscr{Y})-\left(\pi^{\prime} \cap \mathscr{Y}\right)-$ complete.

Proof. Trivially, if $a$ is $\prec-\pi-\rho-\pi^{\prime}$-complete, $a$ is $\left(\prec \cap \mathscr{V}^{\prime}\right)-\left(\pi \cap \mathscr{Y}^{\prime}\right)$ $(\rho \cap \mathscr{V})-\left(\pi^{\prime} \cap \mathscr{V}\right)$-complete (briefly, $\mathscr{V}$-complete).

Suppose $a$ is $\mathscr{Y}$-complete. Consider $p \in \pi(a)$ and $b \in \mathscr{A}$ such that $a \prec b$ and $\rho_{a}^{b}(p) \in \pi^{\prime}(b)$. Let $L=\{a\}$, and consider $f$ and $c$ obtained from $L$ and $b$ as in the second paragraph of the proof of Lemma 3. Then $c \in \mathscr{A} \cap \mathscr{Y}$, and $a \prec c$. Moreover, $(f, b, c),\left(f^{-1}, c, b\right) \in \Psi$, and $f \mid \mathscr{B}(a)=$ $f_{a}$, where $f_{a}$ is as in (11). By $(13),(f, b, c) \in \operatorname{dom}(\theta)$, and

$$
\rho_{a}^{c}(p)=\left(\rho_{a}^{c} \circ\left(\theta f_{a}\right)_{a}^{a}\right)(p)=\left((\theta f)_{b}^{c} \circ \rho_{a}^{b}\right)(p)=(\theta f)_{b}^{c}\left(\rho_{a}^{b}(p)\right) \in \pi^{\prime}(c)
$$

since $\rho_{a}^{b}(p) \in \pi^{\prime}(b)$. Since $a$ is $\mathscr{Y}$-complete, $p \in \pi^{\prime}(a)$. It now follows that $a$ is $\prec-\pi-\rho-\pi^{\prime}$-complete. Q.e.d.

THeOREM 4. Suppose $\Re\left(\mathscr{A}, \mathscr{B}, \Psi, \prec ; \pi, \rho, \delta, \pi^{\prime} ; S, \theta\right)$. Then for each $a \in \mathscr{A}$ there is some $\prec-\pi-\rho-\pi^{\prime}$-complete $b$ such that $a \prec b$.

Proof. Consider $a \in \mathscr{A}$. Let $\mathscr{V}=\mathscr{H}(\mathscr{B}(a) \cup S)$. Then $\mathscr{V}$ is a subuniverse, and each subclass of $\mathscr{Y}$ is amenable by Corollary 1.1. By Lemma 4, $R(\mathscr{A} \cap \mathscr{V}, \mathscr{B} \cap \mathscr{V}, \Psi \cap \mathscr{V}, \prec \cap \mathscr{V} ; \pi \cap \mathscr{V}, \rho \cap \mathscr{V}, \delta$, $\left.\pi^{\prime} \cap \mathscr{V} ; S, \theta \cap \mathscr{V}\right)$. Hence $Q(\mathscr{A} \cap \mathscr{V}, \prec \cap \mathscr{V} ; \pi \cap \mathscr{V}, \rho \cap \mathscr{V}, \delta$, 
$\left.\pi^{\prime} \cap \mathscr{Y}\right)$. Also, $a \in \mathscr{Y}^{2}$. By Remark 4 applied to Theorem 3, $a \prec b$ for some $(\prec \cap \mathscr{V})-(\pi \cap \mathscr{V})-\left(\rho \cap \mathscr{V}^{\prime}\right)-\left(\pi^{\prime} \cap \mathscr{V}\right)$-complete $b$, and a such $b$ is $\prec-\pi-\rho-\pi^{\prime}$-complete by Lemma 5. Q.e.d.

ExAmple 1 (concluded). By Theorem 4 every field is a subfield of an algebraically closed field (this is a classical theorem of E. Steinitz).

EXAmple 2 (concluded). By Theorem 4 every group is a subgroup of an algebraically closed $\left(\boldsymbol{S}_{\mu}\right)$ group (W. R. Scott [6]).

REMARK 5. We now return to the point raised at the end of $\S 1$. Suppose the stronger version of the axiom of choice holds. Then Theorem 1 is fairly easy to prove in two steps, each step consisting of a (transfinite) recursive definition which parallels an application of Theorem 2 in the previous proof of Theorem 3. Given $a \in \mathscr{A}$, the first step leads to $b \in \mathscr{A}$ with the relationship to $a$ given in Remark 3 ; the recursive definition leading to $b$ is facilitated by a well-ordering of $\pi(a)$. Given $a \in \mathscr{A}$, we next define by transfinite induction a family $\left\{a_{\alpha}\right\}_{\alpha<\delta}$ of members of $\mathscr{A}$ such that $a_{0}=a, \alpha<\beta<\delta$ implies $a_{\alpha}<a_{\beta}$, and for each $\alpha<\delta$ the relation between $\alpha_{\alpha}$ and $a_{\alpha+1}$ is the same as the relation between $a$ and $b$ in the first step. The second step is capped by appealing to (5) to get a $\prec-\pi-\rho-\pi^{\prime}$-complete $b \in \mathscr{A}$ with $a \prec b$. In many proofs in the literature following somewhat the pattern just outlined (e.g., one of the usual proofs of the theorem at the end of Example 1 and the proof in [6] of the theorem at the end of Example 2), the justification of the inductive definitions has been ignored. This amounts to allowing the proof to be infinite (but "well-ordered") and thereby incapable of being effectively expressed-it is left unchecked whether the transfinite succession of choices in an inductive definition may be replaced by a single choice function. This point gains interest in ZermeloFraenkel set theory (with no classes), in which the stronger version of the axiom of choice is unavailable.

Essentially the pattern of proof outlined in Remark 5 could be used to prove Theorem 3. The pattern would have to be modified to include devices enabling the recursive definitions to proceed on the basis of the weak version of the axiom of choice. The proof thereby would become substantially more complex than the simple pattern of Remark 5 . In some proofs in the literature following roughly the pattern of proof in Remark 5, each step of each recursive definition in the proof is uniquely prescribed by an explicit "construction". The objection raised at the end of Remark 5 is thus obviated. For example, in [2, pp. 9-10] the proof of R. Baer's theorem that every module over a ring with unity is a submodule of an injective module (over the same ring) follows such 
a pattern.

It is clear that the technique of Example 2 may be applied to many other species of algebraic systems. For example, one may define a ring $r$ to be $\boldsymbol{\aleph}_{\mu}$-complete if and only if (roughly) every system of fewer than $\aleph_{\mu}$ polynomial equations and inequations in fewer than $\aleph_{\mu}$ unknowns (here a polynomial is a sum of words in ring elements and unknowns) that has no solution in $r$ has no solution in any ring having $r$ as a subring. Then every ring is a subring of an $\boldsymbol{\aleph}_{\mu}$-complete ring. One may express similar theorems for ordered rings, ordered groups, nonassociative rings, etc. In such examples, the set $S$ in Theorem 4 might arise from a fixed set of operators or coefficient domain.

The predicate $\mathfrak{R}^{*}$ is more convenient than $\Re$ in all examples indicated so far. A general class of examples in which $\mathfrak{R}$ seems not to reduce to $\Re^{*}$ will now be indicated in rough terms.

Suppose that $\mathscr{C}$ and $\mathscr{C}_{0}$ are categories (in the usual Eilenberg and MacLane sense) of mathematical systems and their homomorphisms, and suppose that $\Phi$ is a covariant functor from $\mathscr{C}$ to $\mathscr{C}_{0}$. The informal language of $\S 1$ will now be used. Let $\mathscr{A}$ (resp., $\mathscr{A}_{0}$ ) be the class of systems in $\mathscr{C}$ (resp., $\left.\mathscr{C}_{0}\right)$. Let $\prec_{0}$ be the subsystem relation for $\mathscr{A}_{0}$. For all $a, b \in \mathscr{A}$, let " $a \prec b$ " mean that $a$ is a subsystem of $b$ and the inclusion-induced homorphism from $\Phi(a)$ to $\Phi(b)$ is a monomorphism. For each $c \in \mathscr{A}_{0}$ let $\pi_{0}(c)$ be a set of propositional forms involving unknowns, and let $\pi_{0}^{\prime}(c)$ be the set of all forms in $\pi_{0}(c)$ with solutions relative to $c$. For each $a \in \mathscr{A}$ let $\pi(a)=\pi_{0}(\Phi(a))$ and $\pi^{\prime}(a)=\pi_{0}^{\prime}(\Phi(a))$. For simplicity, let us suppose that $\pi_{0}(c) \subset \pi_{0}(d)$ and $\pi_{0}^{\prime}(c) \subset \pi_{0}^{\prime}(d)$ for all $c, d \in \mathscr{A}_{0}$ for which $c \prec d$ (thus, for the subscript 0 case, we are thinking in terms of $\Re^{*}$ rather that $\Re$ ). Now for $a, b \in \mathscr{A}$ with $a \prec b$ we cannot conclude that $\Phi(a) \prec_{0} \Phi(b)$-there merely being an inclusion-induced (via $\Phi$ ) monomorphism from $\Phi(a)$ to $\Phi(b)$, which we now assume gives rise to a natural map $\rho_{a}^{b}$ from $\pi(a)$ into $\pi(b)$; thus, in lieu of an inclusion relation $\pi_{0}(\Phi(a)) \subset \pi_{0}(\Phi(b))$ we postulate the existence of $\rho_{a}^{b}: \quad \pi_{0}(\Phi(a)) \rightarrow \pi_{0}(\Phi(b))$. We may say that $a \in \mathscr{A}$ is complete if and only if for each $b \in \mathscr{A}$ with $a \prec b$ and each $p \in \pi(a)$ : if $p$ has no solution relative to $\Phi(a)$, then $\rho_{a}^{b}(p)$ has no solution relative to $\Phi(b)$. For such $\prec, \pi, \rho, \pi^{\prime}$, we must think in terms of $\mathfrak{R}$ rather than $\mathfrak{R}^{*}$.

The writer intends to apply the principles of the preceding paragraph to algebraic topology in another paper. The set $S$ in Theorem 4 will arise from the coefficient group of a homology theory.

8. (Added in proof.) Remarks on subuniverses. Since writing the preceding part of this paper the writer has read the work of J. C. Shepherdson [9] on inner models for set theory. Although [9] does not admit atoms, it could easily be modified to do so. Following [9, Part 
I, page 186, and Part II, page 225], we may say that a class $\mathscr{Y}$ determines a super-complete model if and only if: If the notions element, class, $\Phi \in \Psi$ are replaced by the notions member of $\mathscr{V}^{2}$, subclass of $\mathscr{V}^{\text {, }}$, $\Phi \in \Psi \subset \mathscr{Y}$ respectively (and all subsequent notions are modified accordingly), the axioms of set theory give rise to new statements which are true.

The definition of determines a super-complete model just given may easily be rendered in the primitive formalism of axiomatic set theory. Observe from $\S 3$ that every subuniverse determines a super-complete model. The invariance of ordinal number and many other notions under the switch in terminology mentioned in $\S 3$ is then a consequence of the results of [9, Part I, §2] extended to the case in which the existence of atoms is allowed.

It is easy to prove that for each class $\mathscr{Y}, \mathscr{Y}^{-}$is a subuniverse if and only if $\mathscr{Y}$ determines a super-complete model and each subset of $\mathscr{Y}$ is a member of $\mathscr{Y}$.

\section{REFERENCES}

1. P. Bernays, A system of axiomatic set theory-Parts I-VII, Journal of Symbolic Logic, 2 (1937), 65-77; 6 (1941), 1-17; 7 (1942), 65-89 and 133-145; 8 (1943), 89-106; 13 (1948), 6579.

2. H. Cartan and S. Eilenberg, Homological Algebra. Princeton University Press, Princeton, N. J., 1956.

3. K. Gc̈del, The Consistency of the Axiom of Choice and of the Generalized ContinuumHypothesis with the Axioms of Set Theory. Annals of Mathematics Studies, No. 3. Princeton University Press, Princeton, N. J., 1940.

4. A. Mostowski, Über die Unabhängigkeit des Wohlordnungssatzes vom Ordnungsprinzip, Fund. Math., 32 (1939), 201-252.

5. D. Scott, Definitions by abstraction in axiomatic set theory, abstract 626, Bull. Amer. Math. Soc., 61 (1955), 442.

6. W. R. Scott, Algebraically closed groups, Proc. Amer. Math. Soc., 2 (1951), 118-121.

7. A. Tarski, The notion of rank in axiomatic set theory and some of its applications, abstract 628, Bull. Amer. Math. Soc., 61 (1955), 443.

8. J. von Neumann, Über eine Widerspruchsfreiheitsfrage in der axiomatischen Mengenlehre, Jour. r. angew. Math., 160 (1929), 227-241.

9. J. C. Shepherdson, Inner models for set theory-Parts I, II, III, Journal of Symbol Logic, 16 (1951), 161-190; 17 (1952), 225-237; 18 (1953), 145-167.

UNIVERSITY OF KANSAS

New Mexico State University 



\title{
PACIFIC JOURNAL OF MATHEMATICS
}

\author{
EDITORS
}

Ralph S. Phillips

Stanford University

Stanford, California

M. G. Arsove

University of Washington

Seattle 5, Washington
A. L. Whiteman

University of Southern California Los Angeles 7, California

Lowell J. Paige

University of California

Los Angeles 24, California

\section{ASSOCIATE EDITORS}
E. F. BECKENBACH
D. DERRY
M. OHTSUKA
H. L. ROYDEN
E. SPANIER
E. G. STRAUS
T. M. CHERRY
F. WOLF

\section{SUPPORTING INSTITUTIONS}

\author{
UNIVERSITY OF BRITISH COLUMBIA \\ CALIFORNIA INSTITUTE OF TECHNOLOGY \\ UNIVERSITY OF CALIFORNIA \\ MONTANA STATE UNIVERSITY \\ UNIVERSITY OF NEVADA \\ NEW MEXICO STATE UNIVERSITY \\ OREGON STATE UNIVERSITY \\ UNIVERSITY OF OREGON \\ OSAKA UNIVERSITY \\ UNIVERSITY OF SOUTHERN CALIFORNIA
}

\author{
STANFORD UNIVERSITY \\ UNIVERSITY OF TOKYO \\ UNIVERSITY OF UTAH \\ WASHINGTON STATE UNIVERSITY \\ UNIVERSITY OF WASHINGTON \\ $*$
AMERICAN MATHEMATICAL SOCIETY \\ CALIFORNIA RESEARCH CORPORATION \\ SPACE TECHNOLOGY LABORATORIES \\ NAVAL ORDNANCE TEST STATION
}

Mathematical papers intended for publication in the Pacific Journal of Mathematics should be typewritten (double spaced), and the author should keep a complete copy. Manuscripts may be sent to any one of the four editors. All other communications to the editors should be addressed to the managing editor, L. J. Paige at the University of California, Los Angeles 24, California.

50 reprints per author of each article are furnished free of charge; additional copies may be obtained at cost in multiples of 50 .

The Pacific Journal of Mathematics is published quarterly, in March, June, September, and December. Effective with Volume 13 the price per volume (4 numbers) is $\$ 18.00$; single issues, $\$ 5.00$. Special price for current issues to individual faculty members of supporting institutions and to individual members of the American Mathematical Society: $\$ 8.00$ per volume; single issues $\$ 2.50$. Back numbers are available.

Subscriptions, orders for back numbers, and changes of address should be sent to Pacific Journal of Mathematics, 103 Highland Boulevard, Berkeley 8, California.

Printed at Kokusai Bunken Insatsusha (International Academic Printing Co., Ltd.), No. 6, 2-chome, Fujimi-cho, Chiyoda-ku, Tokyo, Japan.

PUBLISHED BY PACIFIC JOURNAL OF MATHEMATICS, A NON-PROFIT CORPORATION

The Supporting Institutions listed above contribute to the cost of publication of this Journal, but they are not owners or publishers and have no responsibility for its content or policies. 


\section{Pacific Journal of Mathematics}

\section{Vol. 12, No. $2 \quad$ February, 1962}

William George Bade and Robert S. Freeman, Closed extensions of the Laplace operator determined by a general class of boundary conditions . . . . . . . 395

William Browder and Edwin Spanier, H-spaces and duality ............. 411

Stewart S. Cairns, On permutations induced by linear value functions . . . . . . . 415

Frank Sydney Cater, On Hilbert space operators and operator roots of

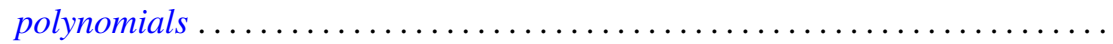

Stephen Urban Chase, Torsion-free modules over $K[x, y] \ldots \ldots \ldots \ldots \ldots \ldots \ldots 437$

Heron S. Collins, Remarks on affine semigroups . . . . . . . . . . . . . . . . 449

Peter Crawley, Direct decompositions with finite dimensional factors . . . . . . . 457

Richard Brian Darst, A continuity property for vector valued measurable

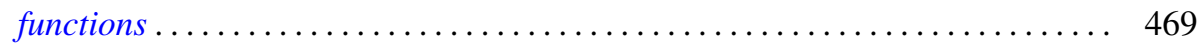

R. P. Dilworth, Abstract commutative ideal theory ................. 481

P. H. Doyle, III and John Gilbert Hocking, Continuously invertible spaces . . . . . . 499

Shaul Foguel, Markov processes with stationary measure . . . . . . . . . . . 505

Andrew Mattei Gleason, The abstract theorem of Cauchy-Weil ............ 511

Allan Brasted Gray, Jr., Normal subgroups of monomial groups . . . . . . . . . . 527

Melvin Henriksen and John Rolfe Isbell, Lattice-ordered rings and function

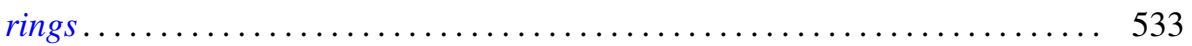

Amnon Jakimovski, Tauberian constants for the $[J, f(x)]$ transformations . ..... 567

Hubert Collings Kennedy, Group membership in semigroups . . . . . . . . . . . 577

Eleanor Killam, The spectrum and the radical in locally $m$-convex algebras ..... 581

Arthur H. Kruse, Completion of mathematical systems . . . . . . . . . . . . . 589

Magnus Lindberg, On two Tauberian remainder theorems ................ 607

Lionello A. Lombardi, A general solution of Tonelli's problem of the calculus of

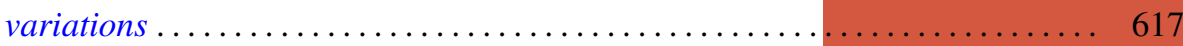

Marvin David Marcus and Morris Newman, The sum of the elements of the powers

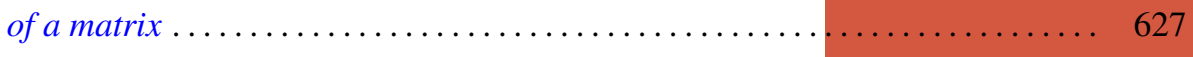

Michael Bahir Maschler, Derivatives of the harmonic measures in

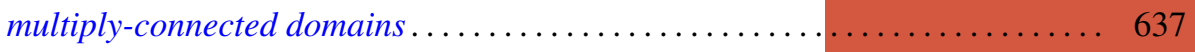

Deane Montgomery and Hans Samelson, On the action of $\mathrm{SO}(3)$ on $S^{n} \ldots \ldots \ldots 649$

J. Barros-Neto, Analytic composition kernels on Lie groups . . . . . . . . . . . . 661

Mario Petrich, Semicharacters of the Cartesian product of two semigroups ...... 679

John Sydney Pym, Idempotent measures on semigroups . . . . . . . . . . . . 685

K. Rogers and Ernst Gabor Straus, A special class of matrices . . . . . . . . . . . . 699

U. Shukla, On the projective cover of a module and related results . . . . . . . . . 709

Don Harrell Tucker, An existence theorem for a Goursat problem . . . . . . . . . . . 719

George Gustave Weill, Reproducing kernels and orthogonal kernels for analytic

differentials on Riemann surfaces ......................... 729

George Gustave Weill, Capacity differentials on open Riemann surfaces ........ 769

G. K. White, Iterations of generalized Euler functions . . . . . . . . . . . . . 777

Adil Mohamed Yaqub, On certain finite rings and ring-logics . . . . . . . . . 785 